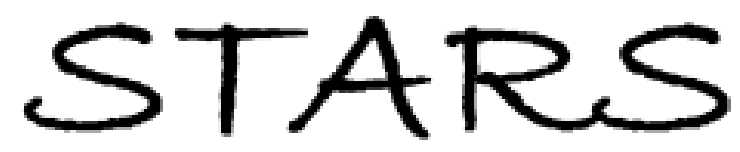

University of Central Florida

STARS

$1-1-2012$

\title{
Cascaded plasmon resonant field enhancement in nanoparticle dimers in the point dipole limit
}

\author{
Seyfollah Toroghi \\ University of Central Florida \\ Pieter G. Kik \\ University of Central Florida
}

Find similar works at: https://stars.library.ucf.edu/facultybib2010

University of Central Florida Libraries http://library.ucf.edu

This Article is brought to you for free and open access by the Faculty Bibliography at STARS. It has been accepted for inclusion in Faculty Bibliography 2010 s by an authorized administrator of STARS. For more information, please contact STARS@ucf.edu.

\section{Recommended Citation}

Toroghi, Seyfollah and Kik, Pieter G., "Cascaded plasmon resonant field enhancement in nanoparticle dimers in the point dipole limit" (2012). Faculty Bibliography 2010s. 3395.

https://stars.library.ucf.edu/facultybib2010/3395

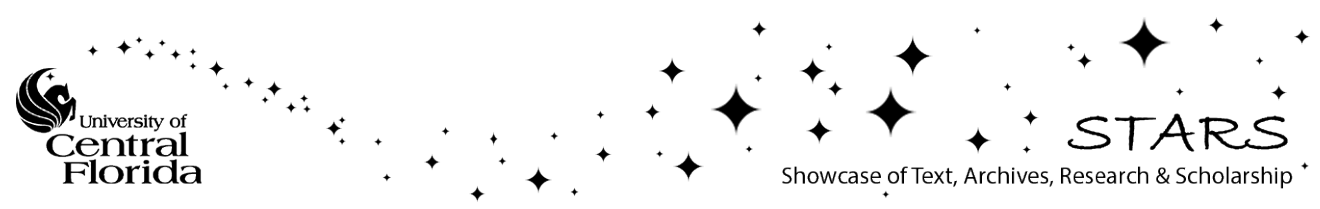




\section{Cascaded plasmon resonant field enhancement in nanoparticle dimers in the point dipole limit}

Cite as: Appl. Phys. Lett. 100, 183105 (2012); https://doi.org/10.1063/1.4707159

Submitted: 13 March 2012 . Accepted: 10 April 2012 . Published Online: 02 May 2012

Seyfollah Toroghi, and Pieter G. Kik

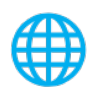

View Online

Export Citation

\section{ARTICLES YOU MAY BE INTERESTED IN}

Cascaded field enhancement in plasmon resonant dimer nanoantennas compatible with twodimensional nanofabrication methods

Applied Physics Letters 101, 013116 (2012); https://doi.org/10.1063/1.4733329

Electromagnetic fields around silver nanoparticles and dimers

The Journal of Chemical Physics 120, 357 (2004); https://doi.org/10.1063/1.1629280

Collective plasmon modes in a compositionally asymmetric nanoparticle dimer

AIP Advances 1, 032134 (2011); https://doi.org/10.1063/1.3628346

\section{Applied Physics Reviews} Now accepting original research 


\title{
Cascaded plasmon resonant field enhancement in nanoparticle dimers in the point dipole limit
}

\author{
Seyfollah Toroghi and Pieter G. Kik ${ }^{\mathrm{a})}$ \\ CREOL, The College of Optics and Photonics, University of Central Florida, 4000 Central Florida Blvd., \\ Orlando, Florida 32816, USA
}

(Received 13 March 2012; accepted 10 April 2012; published online 2 May 2012)

\begin{abstract}
Cascaded field enhancement in silver dimer nanostructures is investigated using a dipole-dipole interaction model. Field enhancement spectra are evaluated as a function of the particle size difference and inter-particle spacing. We observe three distinct regimes of cascaded field enhancement: hindered cascading, multiplicative cascading, and the ultimate cascading limit, depending on the dimer interaction strength. Multiplicative cascading at small inter-particle spacing leads to analytic expressions for the ultimate internal and external field enhancement factors. For silver dimers in a host with index 1.5, we obtain a maximum internal field enhancement of $2.9 \times 10^{3}$, a factor of 75 larger than that of an isolated silver nanoparticle. (C) 2012 American Institute of Physics. [http://dx.doi.org/10.1063/1.4707159]
\end{abstract}

It has been demonstrated that a self-similar chain of plasmon resonant metal nanoparticles can produce extremely large field enhancement factors due to a phenomenon called "cascaded plasmon resonance." This effect occurs when small particles are driven by the enhanced local field of a nearby larger particle, leading to extreme field confinement. Cascaded plasmon resonance could enable a broad variety of enhanced optical effects, including surface enhanced Raman scattering $^{2}$ (SERS), enhanced nonlinear optical response, ${ }^{3,4}$ and enhanced stimulated emission. ${ }^{5}$ Due to the substantial potential impact on optical phenomena and applications, several studies have investigated plasmon cascading both experimentally and theoretically. In recent studies, Sun et al. presented an analytical model that describes the field enhancement in coupled particle systems, ${ }^{6,7}$ focusing on the external field enhancement that could be achieved in the presence of multipolar interactions. This work demonstrated the existence of a maximum external field enhancement and showed that multipoles can play a role in the enhancement spectra. In the present study, we systematically investigate the evolution of cascaded field enhancement in the point dipole limit. This approach allows us to highlight the role of the mutual interaction strength in the coupled system and to identify distinct interaction regimes for asymmetric silver nanoparticle dimers as a function of particle size difference and inter-particle spacing. Figure 1(a) shows a schematic of the type of structure under investigation as well as calculated internal field enhancement spectra using the dipole-dipole interaction model (solid line) and using full electromagnetic simulation (dashed line). The dipole interaction model closely matches the numerical simulation results despite the absence of multipolar interactions in the former. It should be noted that multipolar interactions can only be ignored in the case of large particle size differences or weak inter-particle interaction, as discussed in more detail below. Figure 1(b) shows the corresponding spatial distribution of the electric field enhancement at the plasmon resonance wavelength,

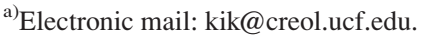

demonstrating the extremely localized nature of the field enhancement. The presented model allows for the rapid evaluation of the particle size differences and inter-particle spacing required in order to achieve the ultimate cascaded field enhancement.

Each nanosphere is modeled as a point dipole with an effective polarizability $\alpha_{i}=3 \varepsilon_{0} \varepsilon_{h} V_{i}\left(\varepsilon_{m}-\varepsilon_{h}\right) /\left(\varepsilon_{m}+2 \varepsilon_{h}\right)$ where $V_{i}$ is the volume of nanosphere $i$, and $\varepsilon_{m}$ and $\varepsilon_{h}$ are the dielectric function of the metal and the host, respectively. The dipole moment of nanosphere $i$ at position $\boldsymbol{r}_{i}$ is $\boldsymbol{p}_{i}\left(\boldsymbol{r}_{i}\right)=\alpha_{i}$ $\boldsymbol{E}_{l o c}\left(\boldsymbol{r}_{i}\right)$ where $\boldsymbol{E}_{l o c}\left(\boldsymbol{r}_{i}\right)$ is the sum of the incident field $\boldsymbol{E}_{0}\left(\boldsymbol{r}_{i}\right)$ and any additional electric fields at location $\boldsymbol{r}_{i}$ originating from neighboring dipoles, leading to $\boldsymbol{E}_{l o c}\left(\boldsymbol{r}_{i}\right)=\boldsymbol{E}_{0}\left(\boldsymbol{r}_{i}\right)-\sum \boldsymbol{A}_{\mathrm{ij}}$ $\boldsymbol{p}_{j}$ with $\boldsymbol{A}_{\mathbf{i j}}$ the dipole-dipole interaction matrix. For a dimer system illuminated with light polarized along the dimer axis, $A_{12}$ and $A_{21}$ are equal and given by

$$
A_{12}=A_{21}=\frac{e^{i k d}}{2 \pi \varepsilon_{0} \varepsilon_{h}}\left(\frac{i k}{d^{2}}-\frac{1}{d^{3}}\right),
$$

where $\mathrm{d}$ is the distance between two dipoles and $k$ is the wavevector in the host medium. The dipole moment of each nanoparticle is thus given by

$$
\begin{aligned}
& p_{1}=\alpha_{1}\left[E_{0}-A_{12} p_{2}\right], \\
& p_{2}=\alpha_{2}\left[E_{0}-A_{21} p_{1}\right] .
\end{aligned}
$$
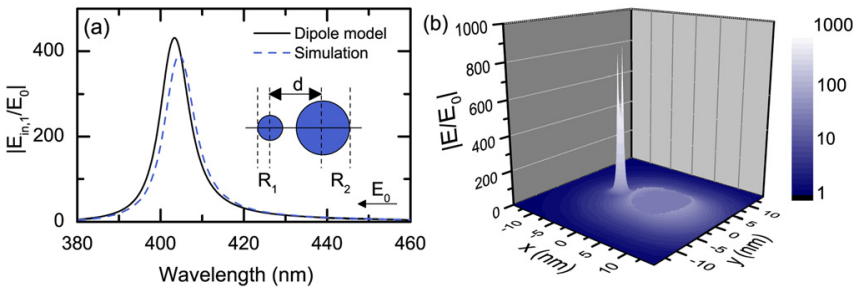

FIG. 1. (a) Field enhancement spectra in the smaller of two particles in a silver dimer with particle radii of $0.5 \mathrm{~nm}$ and $5 \mathrm{~nm}$ at a center-to-center spacing of $9 \mathrm{~nm}$ calculated using a dipole-dipole interaction model (solid line) and using full electromagnetic simulation (dashed line). (b) Simulated electric field enhancement distribution around the same structure at a wavelength of $404 \mathrm{~nm}$. 
Solving Eq. (2) for particle 1 leads to

$$
p_{1}=\alpha_{1} \frac{1-\alpha_{2} A_{12}}{1-\alpha_{1} \alpha_{2} A_{12}^{2}} E_{0}
$$

An analogous expression for particle 2 can be obtained. The term $\alpha_{1} \alpha_{2} A_{12}^{2}$ represents a coupling parameter that describes the dipole interaction strength. Using the known frequencydependent polarizability $\alpha(\omega)$ of the individual particles, Eq. (3) predicts the frequency-dependent dipole moment of particle 1. Taking Eq. (3) and using $E_{l o c, l}=p_{1} / \alpha_{1}$, we obtain $E_{l o c, l}$ which in turn allows us to calculate the internal electric field enhancement factor $g_{i n, l}=E_{i n, l} / E_{O}$ in particle 1 by applying the relation $E_{\text {in }} / E_{l o c}=3 \varepsilon_{h} /\left(\varepsilon_{m}+2 \varepsilon_{h}\right)$

$$
g_{i n, 1}=\frac{E_{i n, 1}}{E_{0}}=\frac{3 \varepsilon_{h}}{\varepsilon_{m}+2 \varepsilon_{h}} \frac{1-\alpha_{2} A_{12}}{1-\alpha_{1} \alpha_{2} A_{12}^{2}} .
$$

The internal field enhancement is important in applications such as plasmon enhanced nonlinear absorption, ${ }^{4}$ where a large part of the nonlinear response is due to the modified optical response of the metal. However, one can easily obtain the corresponding external electric field enhancement factor $g_{\text {out }}$ at the surface of particle 1 along the incident field polarization by replacing the term $3 \varepsilon_{h}$ in Eq. (4) by $3 \varepsilon_{m}$.

Figure 2 shows the internal field enhancement spectra, $\left|g_{i n}\right|=\left|E_{i n} / E_{0}\right|$, in each particle for four silver nanoparticle dimers with different volume ratios, $V_{2} / V_{1}$, at a fixed centerto-center separation $\mathrm{d}=15 \mathrm{~nm}$. The radius of the small nanoparticle is varied while that of the large nanoparticle is kept constant at $5 \mathrm{~nm}$. The particles are embedded in a host with $\varepsilon_{h}=2.25$, and literature data were used for the dielectric function of silver. ${ }^{8}$

Figures 2(a) and 2(b) show the field enhancement in the center of the left and right particle, respectively, for a dimer of particles with identical volume. For comparison, Fig. 2(b)

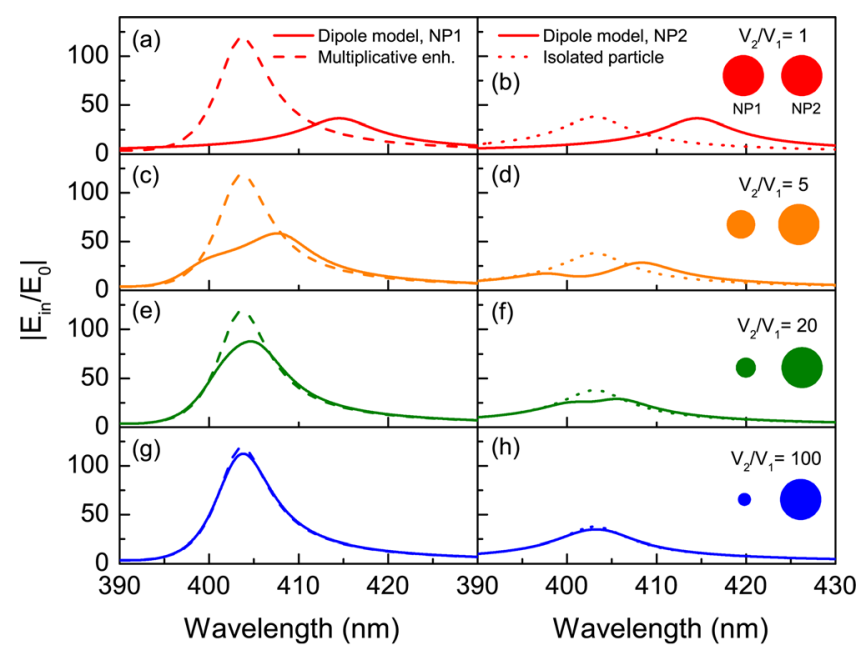

FIG. 2. Internal field enhancement spectra for silver nanoparticle dimers at a constant center-to-center distance of $15 \mathrm{~nm}$, for the small particle (left panels) and the large particle (right panels) for volume ratios $V_{2} / V_{1}=1,5,20$, and 100 as indicated in the insets. The dashed lines (left panels) show multiplicative cascaded field enhancement while the dotted lines (right panel) show the analytical single particle field enhancement. includes the internal field enhancement $\left|g_{\text {in,iso }}\right|$ of an isolated silver nanosphere (dotted line). The maximum field enhancement values in the dimer are similar to those of the isolated sphere, but occur at longer wavelength due to near-field coupling between the particles. ${ }^{9}$ The enhancement factors are much weaker than expected for cascaded field enhancement: in the simplest interpretation of cascading, particle 1 is driven by a modified local field $E_{\mathrm{loc}, 1}$, leading to a predicted multiplicative field enhancement magnitude of $\left|g_{\text {in,iso }} \times E_{\text {loc, }, 1}\right|$. This response is shown in Fig. 2(a) (dashed line), showing a peak enhancement of 119 , compared to an actual peak enhancement of 36.5. Figures 2(c) and 2(d) show the results for a dimer with a volume ratio of 5 . The field enhancement in the large particle shows two resonance peaks, with the lowfrequency resonance corresponding to a symmetric mode where the dipole moments in both particles oscillate in-phase (phase data not shown), and the high-frequency resonance corresponding to an anti-symmetric mode where the dipole moments in both particles oscillate in anti-phase. ${ }^{10}$ At this larger volume ratio, the peak field enhancement in the small particle has increased, but is still substantially lower than the multiplicative cascading response. Figures 2(e) and 2(f) show the results for a volume ratio of 20 , showing less pronounced splitting, indicative of a reduced interaction. Note that the internal field enhancement in the small particle approaches the multiplicative cascading response. Finally, Figs. 2(g) and 2(h) show the results for a volume ratio of 100 . No clear frequency splitting is observed, the response of the large nanoparticle is almost identical to that of an isolated nanoparticle, and the internal field enhancement of the small particle is virtually identical to the calculated multiplicative cascading response. These results demonstrate that multiplicative cascading requires weak mutual interaction, corresponding to $\alpha_{1} \alpha_{2} A_{12}^{2} \ll 1$ in Eq. (4). For strong interaction, we observe reduced field enhancement, a coupling regime that we will call hindered cascading.

Figure 3 shows the field enhancement spectra in silver nanoparticle dimers with $V_{2} / V_{1}=100$ for different center-tocenter separations. Figures 3(a) and 3(b) show the results for a center-to-center separation of $40 \mathrm{~nm}$ for the small and large particle, respectively. At this separation, the magnitude of the internal field enhancement of the large nanoparticle is approximately equal to that of an isolated nanoparticle $\left|g_{\text {in,iso }}\right|$ (Fig. 3(b), dotted line). The field enhancement of the small particle is a factor $\sim 1.5$ larger than observed in the large particle, and its spectrum is nearly identical to the multiplicative cascading result (Fig. 3(a), dashed line), indicating that the particles are weakly coupled. Figures 3(c) and 3(d) show the results for a center-to-center separation of $15 \mathrm{~nm}$. The smaller separation leads to an increased field enhancement in the small particle due to the larger near field provided by the large particle at this distance, while the internal field enhancement of the small particle is close to the multiplicative field enhancement result. At a center-to-center separation of $12 \mathrm{~nm}$ (Figs. 3(e) and 3(f)), clear splitting is observed in the field enhancement spectra, while the peak value of the field enhancement of the small nanoparticle is approximately 200 , significantly below the predicted multiplicative field enhancement. Finally, for a centerto-center separation of $10 \mathrm{~nm}$ (Figs. $3(\mathrm{~g})$ and 3(h)), a large mode splitting is observed while the maximum internal field 


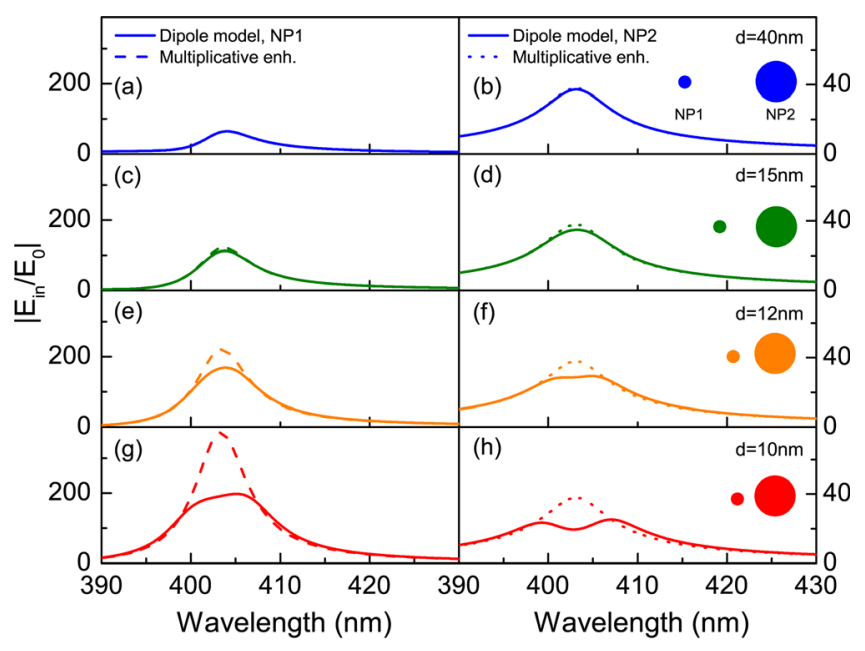

FIG. 3. Internal field enhancement spectra for silver nanoparticle dimers at a fixed volume ratio of 100 for the small particle (left panels) and the large particle (right panels) for inter-particle separations of $\mathrm{d}=10 \mathrm{~nm}, 12 \mathrm{~nm}$, $15 \mathrm{~nm}$, and $40 \mathrm{~nm}$ as indicated in the insets. The dashed lines (left panels) show multiplicative cascaded field enhancement while the dotted lines (right panel) show the analytical single particle field enhancement.

enhancement of the small nanoparticle remains approximately the same, with a value well below the theoretical multiplicative field enhancement due to the strong mutual interaction.

The observations in Figs. 2 and 3 indicate the existence of an ultimate cascading limit, corresponding to a situation in which the smaller particle is sufficiently close to the large nanoparticle to experience its maximum external field enhancement of $\left|3 \varepsilon_{\mathrm{m}}\left(\omega_{\mathrm{LSP}}\right) /\left(\varepsilon_{\mathrm{m}}\left(\omega_{\mathrm{LSP}}\right)+2 \varepsilon_{\mathrm{h}}\right)\right|$, with $\omega_{\mathrm{LSP}}$ the localized surface plasmon resonance frequency of an isolated nanosphere. In this case, multiplicative cascading would produce an ultimate internal field enhancement of magnitude $\left|g_{\text {ucl,in }}\right|$ $=\left|g_{1, \text { in }}\left(\omega_{L S P}\right) \times 3 \varepsilon_{\mathrm{m}}\left(\omega_{\mathrm{LSP}}\right) /\left(\varepsilon_{\mathrm{m}}\left(\omega_{\mathrm{LSP}}\right)+2 \varepsilon_{\mathrm{h}}\right)\right|=2\left(3 \varepsilon_{\mathrm{h}} / \operatorname{Im}\left(\varepsilon_{\mathrm{m}}\right)\right)^{2}$. Similarly, we can obtain an ultimate external cascaded field enhancement limit near the small particle given by $\left|g_{\text {ucl,ext }}\right|=2$ $\left|g_{u c l, i n}\right|$. In the following, we investigate the evolution of the cascaded field enhancement as a function of geometry and compare the obtained values to the theoretical ultimate cascading limit.

Figure 4 shows the maximum internal field enhancement in silver nanoparticle dimer as a function of center-to-center distance and particle radius calculated using the dipole interaction model. The radius of the large nanoparticle is held at $5 \mathrm{~nm}$ while the radius of the small particle $R_{1}$ is varied. For small size difference (large $\mathrm{R}_{1}$ ) or large inter-particle spacing, low field enhancement values are observed. For most geometries, the field enhancement exceeds that of an isolated silver nanoparticle (black dotted contour line), with the exception of dimers with small volume ratio or large interparticle spacing. The former exception is due to a significant redshift in the main resonance frequency (see Figs. 2(a) and 2(b)), which is accompanied by an increase of the imaginary part of the Au dielectric function. The latter exception is due to the oscillatory nature of the dipole interaction term in Eq. (1), in particular the term $e^{i k d}$, allowing for both constructive and destructive interaction between the particles in the dimer depending on the inter-particle spacing. The largest field enhancement values are observed at small spacing and large size difference. To indicate the transition from hindered cas-

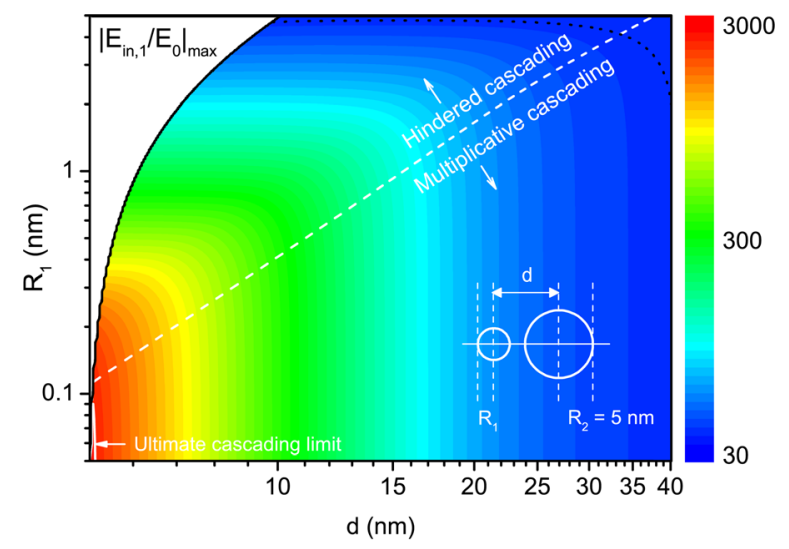

FIG. 4. Maximum internal field enhancement in silver nanoparticle dimers calculated by a dipole interaction model. The dotted black line indicates the field enhancement of an isolated Ag nanosphere. The white dashed line separates the hindered and multiplicative cascading regimes. The white solid line shows field enhancement values that are within $15 \%$ of the analytical ultimate cascading limit.

cading to multiplicative cascading, the white dashed line shows geometries that produce a field enhancement that is $95 \%$ of the multiplicative cascaded field enhancement. Note that multiplicative cascaded field enhancement requires weak coupling, which can be achieved either for large separation, or for small separation and large size difference. Only for extremely large volume ratios and small separation, field enhancements are observed that approach the calculated ultimate cascading limit of $\left|g_{\text {ucl }}\right|=2.9 \times 10^{3}$ for these materials. The corresponding maximum external field enhancement is $5.8 \times 10^{3}$, which is a factor $\sim 19$ larger than that calculated for the asymmetric gold dimers studied in Ref. 7 due to the higher surface plasmon damping in gold and the inclusion of radiative damping in Ref. 7.

The white solid contour shows the geometries in which the field enhancement is $85 \%$ of the ultimate cascading limit. It should be pointed out that the point dipole analysis presented here neglects surface scattering, which is known to introduce significant damping at the sizes considered here, and consequently reduced field enhancement factors are expected in experiments on similarly sized particles.

The results shown in Fig. 4 have several implications. First, for most geometries shown, asymmetric nanoparticle dimers produce field enhancement factors that significantly exceed those of isolated particles, even in the case of hindered cascading. Second, approaching the ultimate cascading limit to within $15 \%$ requires extremely large volume ratios, providing a significant challenge for experiments aimed at demonstrating this limit. Third, reaching the ultimate cascading limit requires extremely small edge-to-edge spacing. Finally, it should be pointed out that the dipole interaction model does not include multipolar plasmon modes, which are expected to occur for small inter-particle spacing at intermediate volume ratios. However, for coupling conditions that lead to large near-multiplicative enhancement factors, the mutual interaction is necessarily weak. Since multipolar modes on these particles are predominantly excited due to the inhomogeneous near-field from neighboring particles, weak interaction implies the relatively weak excitation of multipolar modes. Consequently, our simplified model provides surprisingly accurate results especially for systems that 
produce large field enhancement. To illustrate this point, Fig. 1(a) includes internal field enhancement spectra $\left|g_{i n, 1}\right|$ calculated using the point dipole interaction model for a dimer with volume ratio $1000, R_{1}=0.5 \mathrm{~nm}, R_{2}=5 \mathrm{~nm}$, and $\mathrm{d}=9 \mathrm{~nm}$, and using full numerical simulation. ${ }^{11}$ The results show remarkable agreement and demonstrate the usefulness of the dipole model even for finite size structures in the presence of significant cascading under weak mutual interaction.

In summary, a dipole-dipole interaction model was used to evaluate cascaded field enhancement in asymmetric silver nanosphere dimers. The structures were shown to enable a multiplicative cascaded field enhancement of a factor $2.9 \times 10^{3}$ for dimers with large size difference and small inter-particle spacing. The evolution of the field enhancement as a function of particle volume ratio and spacing was analyzed. In the limit of weak-mutual coupling, analytical formulas were derived for the ultimate internal and external field enhancement factors in coupled nanosphere dimers.
This work was supported by the National Science Foundation (CAREER Award No. ECCS-0644228).

${ }^{1}$ K. Li, M. I. Stockman, and D. J. Bergman, Phys. Rev. Lett. 91(22), 227402 (2003).

${ }^{2}$ V. G. Kravets, G. Zoriniants, C. P. Burrows, F. Schedin, C. Casiraghi, P. Klar, A. K. Geim, W. L. Barnes, and A. N. Grigorenko, Phys. Rev. Lett. 105(24), 246806 (2010).

${ }^{3}$ K. Li, M. I. Stockman, and D. J. Bergman, Phys. Rev. B 72(15), 153401 (2005).

${ }^{4}$ S. Toroghi and P. G. Kik, Phys. Rev. B 85(4), 045432 (2012).

${ }^{5}$ K. R. Li, X. T. Li, M. I. Stockman, and D. J. Bergman, Phys. Rev. B 71(11), 115409 (2005).

${ }^{6}$ G. Sun, J. B. Khurgin, and A. Bratkovsky, Phys. Rev. B 84(4), 045415 (2011).

${ }^{7}$ G. Sun and J. B. Khurgin, Appl. Phys. Lett. 98(15), 153115 (2011).

${ }^{8}$ P. B. Johnson and R. W. Christy, Phys. Rev. B 6(12), 4370-4379 (1972).

${ }^{9}$ S. A. Maier, M. L. Brongersma, P. G. Kik, and H. A. Atwater, Phys. Rev. B 65(19), 193408 (2002).

${ }^{10}$ S. Toroghi and P. G. Kik, Proc. SPIE 8054, 80540E (2011).

${ }^{11}$ Microwave Studio, Computer Simulation Technology, Darmstadt, Germany, 2011. 